\title{
Interaction of terahertz radiation with tissue phantoms: numerical and experimental studies
}

\author{
O. A. Smolyanskaya ${ }^{1}$, Q. Cassar ${ }^{2}$, M. S. Kulya ${ }^{1}$, N. V. Petrov ${ }^{1}$, K. I. Zaytsev ${ }^{3,4}$, \\ A. I. Lepeshkin ${ }^{1}$, J.-P. Guillet ${ }^{2}$, P. Mounaix ${ }^{2}$, V. V. Tuchin ${ }^{1,5,6}$ \\ ${ }^{1}$ ITMO University, Saint-Petersburg, Russia, smolyanskaya@corp.ifmo.ru \\ ${ }^{2}$ Bordeaux University, IMS Laboratory, Bordeaux, France \\ ${ }^{3}$ Bauman Moscow State Technical University, Moscow, Russia \\ ${ }^{4}$ Prokhorov General Physics Institute of the RAS, Moscow, Russia \\ ${ }^{5}$ Saratov State University, Saratov, Russia \\ ${ }^{6}$ Institute of Precision Mechanics and Control of the RAS, Saratov, Russia
}

\section{Introduction}

New biomedical devices require test objects to check their performance and periodic calibration to monitor the system efficiency over the time. A phantom is a bio-like object mimicking the intrinsic real-tissue properties that serves as a test object. A variety of phantoms are commercially available. Phantoms are already used in spectroscopy within various spectral range. However, currently, the $\mathrm{THz}$ frequency range suffers from a lack of investigations in this direction. Only the first steps have been performed [1]. In the present work, we investigate interaction of submillimeter radiation with the breast-mimicking and skin-mimicking phantoms. Results for breastmimicking phantom were compared with numerical models based on a double-Debye model of the dielectric permittivity for different content of fat, fibrous and cancerous tissue. The wave front propagation using the angular spectrum representation was used to simulate the theoretical response of skinmimicking phantoms.

\section{Model of wavefront propagation}

Electromagnetic fields can be represented in various ways. The angular spectrum representation is a numerical technique describing optical fields in a homogeneous media [2]. Optical fields are described as a superposition of plane waves and evanescent waves. A useful approach to describe optical field diffraction is to conduct the Fourier analysis at a given plane so that the different Fourier components of the field distribution are identified as plane waves propagating away from that plane in different directions. Thus taking less computation time for numerical reconstruction. This technique is preferable for the analysis of light diffraction by histological slides [3]. Angular spectrum representation consists of the following stages: (i) the representation of the field through the angular spectrum of 2D waves (Eq.1); (ii) multiplication by the transfer function that contains a complex refractive index of the object (Eq.2); (iii) the back transition from plane waves to the wave field in the calculated z-plane (Eq.3).

$$
\begin{aligned}
& C\left(f_{x}, f_{y}, v\right)=\int_{-\infty}^{\infty} \int_{-\infty}^{\infty} G(\tilde{x}, \tilde{y}, v)_{z=0} \exp \left(-2 \pi i\left(\tilde{x} f_{x}+\tilde{y} f_{y}\right)\right) d \tilde{x} d \tilde{y} \\
& g_{x, y}\left(f_{x}, f_{y}, v, z\right)=C\left(f_{x}, f_{y}, v\right) \exp \left(-i \frac{2 \pi v n(v)}{c} \sqrt{1-\left(\frac{f_{x} \cdot c}{v n(v)}\right)^{2}-\left(\frac{f_{y} \cdot c}{v n(v)}\right)^{2}} \cdot z\right) \\
& G(x, y, v, z)=\int_{-\infty}^{\infty} \int_{-\infty}^{\infty} g_{x, y, z}\left(f_{x}, f_{y}, v, z\right) \exp \left(2 \pi i\left(x f_{x}+y f_{y}\right)\right) d f_{x} d f_{y},
\end{aligned}
$$

where $^{n(\tilde{x}, \dot{y}, v)=n_{r e}(\tilde{x}, \tilde{y}, v)+i \cdot n_{i m}(\tilde{x}, \tilde{y}, v)}$ is the spatial and frequency distribution of the complex index of refraction.

\section{Materials and methods}

a. Sample preparation

We have used three-component phantoms made of fat, protein and water of different content. Vegetable oil $(10-75 \%)$, soya $(13-75 \%)$ and water $(0-70 \%)$ were homogenized to generate an emulsion. Then, the phantoms were deposited into vacuum packages.

\section{b. THz-Spectrometer}

The commercially available TPS 4000 (Teraview Ltd, UK) spectrometer working in reflection mode was used. The spectral range of the system is from 0.06 to 4.50 THz. The entire system was under an air-dried dome to limit the interaction of water molecules with generated pulses. Samples were mounted on a sapphire substrate during measurement. The sapphire cut was chosen not to exhibit a birefringence that would disrupt results of measurements [4]. Each sample was measured 5 times, independently, i.e. the sample was removed and replaced again into system.

\section{c. Numerical modelling}

A double-Debye model of the dielectric permittivity was used to compare optical properties of the threecomponent phantoms and some biological tissue (adipose, fibrous and cancerous) taken from our previous paper [5].

\section{Results and discussion}

The propagation dynamics of the pulsed $\mathrm{THz}$ radiation through a skin sample was numerically simulated in the temporal and spectral domains. The index of refraction, 
$n_{\mathrm{re}}$, absorption coefficient, $\alpha$, as well as real, $\varepsilon_{\mathrm{re}}$, and imaginary, $\varepsilon_{\mathrm{im}}$, part of dielectric permittivity was calculated from the experimentally obtained data. Figure 1a depicts the time-domain spatial evolution of the $\mathrm{THz}$ pulse. Agreement between measured and simulated $\mathrm{THz}$ waveforms is confirmed by the comparison shown in Figure 1b.
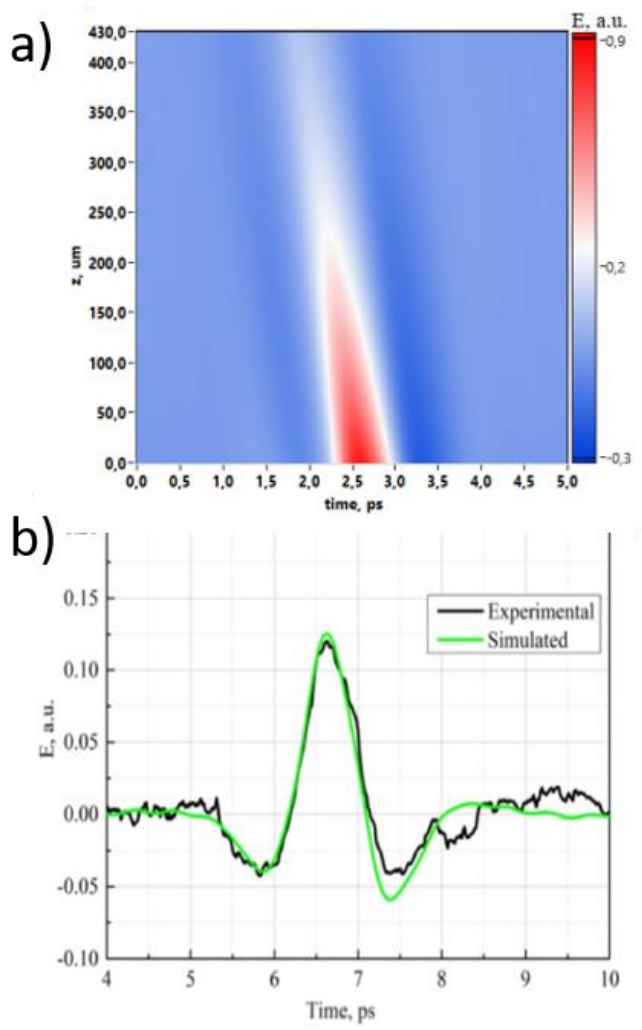

Fig. 1. Experimental and numerically simulated $\mathrm{THz}$ pulses propagated through a skin-mimicking sample. (a) $\mathrm{THz}$ spatial distribution. (b) THz waveforms.

In Figure 2 the spectral dependences of the absorption coefficient of the three-component phantoms consisting of fat, protein and water are depicted. It is shown that within the spectral window, the absorption coefficient of phantoms containing water exceeds the absorption coefficient of phantoms free of water. This is due to the fact, that water molecules, which strongly absorb $\mathrm{THz}$ radiations, are replaced by low absorbing components (fat and protein). Our results were compared with simulated signals of adipose, fibrous and cancerous tissues. taken from our previous paper [5].

\section{Conclusion}

To overcome the lack of knowledge on tissuemimicking phantoms within the submillimeter spectral range, we conducted preliminary studies on skin- and breast tissue-mimicking samples. Simulations performed for skin-mimicking phantoms

have match the experimental spectral data. Thus demonstrating advantages of the technique used in this

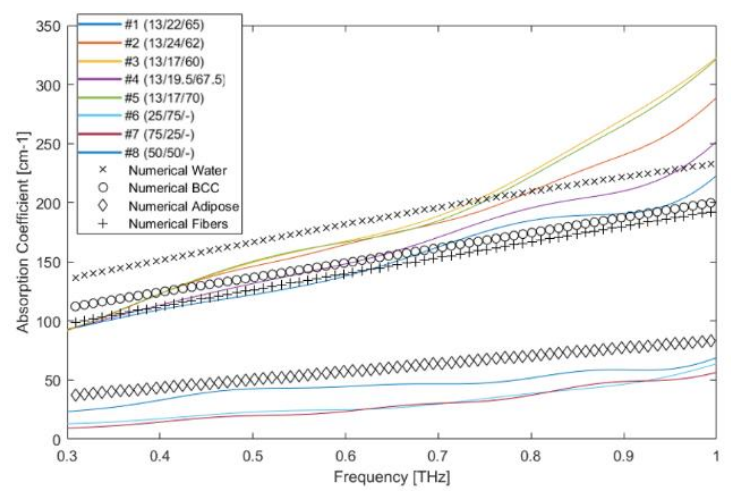

Fig. 2. Spectral dependences of the absorption coefficient of the three-component phantoms consisting of fat, protein and water in the $\mathrm{THz}$ frequency range (solid-colored lines) versus simulated signal of water and purely adipose, fibrous and cancerous tissue types.

study. The absorption coefficient spectral dependences extracted from measurements for breast-mimicking phantom, are clearly differentiated into two distinct groups. One for phantoms containing water and those being water-free. Such a discrimination is also shown via the double-Debye model of the dielectric permittivity. Thus, finding of a proper composition of tissue phantom is possible by an appropriate matching of model and experimental data. Deeper investigations are however still required to fulfill a set of phantoms that could be used to perform tissue measurement within the THz-range. Such a set would be profitable to design new medical $\mathrm{THz}$-devices without human biopsy.

\section{Acknowledgments}

The reported study was funded by RFBR and CNRS according to the research project №18-51-16002, and by the Government of the Russian Federation (Grant 08-08).

\section{References}

1. E Liakhov, O Smolyanskaya, A Popov, E Odlyanitskiy, $N$ Balbekin, $M$ Khodzitsky, Fabrication and characterization of biotissue-mimicking phantoms in the $\mathrm{THz}$ frequency range // Journal of Physics: Conference Series. 2016. No. 735. P. 012080.

2. Petrov, N. V., Kulya, M. S., Tsypkin, A. N., Bespalov, V. G., \& Gorodetsky, A. Application of terahertz pulse time-domain holography for phase imaging // IEEE Transactions on Terahertz Science and Technology, 2016, V. 6, No. 3, P. 464-472.

3. O. A. Smolyanskaya, I. J. Schelkanova, M. S. Kulya, E. L. Odlyanitskiy, I. S. Goryachev, A. N. Tcypkin, Ya V. Grachev, Ya G. Toropova, and V. V. Tuchin // Glycerol dehydration of native and diabetic animal tissues studied by THz-TDS and NMR methods // Biomedical Optics Express, 2018, V. 9, No. 3, P. 1198-1215.

4. Q. Cassar, A. Al-Ibadi, la. Mavarani, Ph. Hillger, J. Grzyb, G. Macgrogan, Th. Zimmer, U.R. Pfeiffer, J.-P. Guillet, P. Mounaix Pilot study of freshly excised breast tissue response in the 300-600 GHz range // Biomed Opt Express. 2018. V. 9 No. 7. P. 2930-2942.

5. A. J. Fitzgerald, E. Pickwell-MacPherson, and V. P. Wallace, Use of finite difference time domain simulations and Debye theory for modelling the terahertz reflection response of normal and tumour breast tissue // Plos One 9, 1-9 (2014). 